OPEN ACCESS

Edited by:

Hongyu Zhang,

Tsinghua University, China

Reviewed by:

Shanhua Qian,

Jiangnan University, China

Liguo Qin,

Xi'an Jiaotong University, China

Yu Yan,

University of Science and Technology

Beijing, China

${ }^{*}$ Correspondence:

Zikai Hua

eddie_hua@shu.edu.cn

Specialty section

This article was submitted to

Tribology,

a section of the journal

Frontiers in Mechanical Engineering

Received: 04 December 2018

Accepted: 13 March 2019

Published: 05 April 2019

Citation:

Hua Z, Dou P, Jia H, Tang F, Wang $X$

Xiong $X$, Gao L, Huang $X$ and Jin $Z$

(2019) Wear Test Apparatus for

Friction and Wear Evaluation Hip

Prostheses. Front. Mech. Eng. 5:12.

doi: 10.3389/fmech.2019.00012

\section{Wear Test Apparatus for Friction and Wear Evaluation Hip Prostheses}

\author{
Zikai Hua ${ }^{1 *}$, Pingchuan Dou ${ }^{1}$, Haili Jia ${ }^{1}$, Fei Tang ${ }^{1}$, Xiaojing Wang ${ }^{1}$, Xin Xiong ${ }^{1}$, \\ Leiming $\mathrm{GaO}^{2}$, Xiuling Huang ${ }^{1}$ and Zhongmin $\mathrm{Jin}^{3}$ \\ ${ }^{1}$ Orthotek Laboratory, School of Mechatronics Engineering and Automation, Shanghai University, Shanghai, China, \\ ${ }^{2}$ Department of Aeronautics, Imperial College London, London, United Kingdom, ${ }^{3}$ School of Mechanical Engineering, \\ University of Leeds, Leeds, United Kingdom
}

Wear is a key factor affecting the survivorship of artificial hip joints. Joint wear testers, as called "simulators," are developed to simulate the motions and force of human joints. By using simulators, in vitro wear tests could be performed for artificial joints. This paper introduces a new hip joint simulator developed for the biotribological investigation of artificial hip joints. Friction force measuring module has been set up and assembled to monitor the friction force during the wear tests. Validation tests have been performed using two kinds of wear couples of $28 \mathrm{~mm}$ CoCrMo femoral heads, against conventional and X-linked ultra-high molecular weight polyethylene (UHMWPE) acetabular cups. The hip joint simulator is a precise and reliable method, both for the wear evaluation and biotribological investigation of hip prostheses.

Keywords: wear, hip joint, simulator, friction, UHMWPE

\section{INTRODUCTION}

Total Hip Replacement (THR) surgeries are considered as an efficient method to treat degenerative joint diseases. However, the survivorship of THR decreases dramatically after 15 years of use (Ferguson et al., 2018). A typical clinical wear rate of ultra-high molecular weight polyethylene (UHMWPE) cups, of 100 300 $\mu \mathrm{m} / \mathrm{year}$, will lead to loosening, inflammation and ultimately failure (McKellop et al., 1995). Efforts are seriously needed to prolong the survival of the hip prostheses, which is important for optimizing patient care and medical costs (Chu, 2015). Consequently, the bio-tribological performance of orthopedic implants has been gaining increasing attention. The tribological evaluation of such kinds of implants is regarded as an essential evaluation of hip prostheses.

Hip joint wear testers, as called hip joint simulator, is developed for the wear evaluation of hip prostheses during a specified duration, under body-simulated conditions referring to the dynamic and kinematic characteristics of human hip joint (Cheng and Shan, 2012). Wear rates and particles in the hip simulator studies are similar to those observed in vivo experience (Hua and Zhang, 2008; Saikko and Shen, 2010; Oliveira et al., 2013; Wang et al., 2013). Currently, ISO organization has published several standards on the wear tests of hip prostheses (ISO, 2009, 2014, 2016).

For a 5-million-cycle hip simulator wear test, the duration usually lasts over 3 months. And the wear rates, obtained by interval measuring, are regarded as the most important results in such kinds of tests. Online information like friction torque and lubricant performance cannot be well-studied. Therefore, besides the material removal, other properties of the prostheses articulating surfaces are needed to understand the dynamic performance during the wear tests. Frictional force monitoring is a valid complementary factor to represent the true tribological behavior. However, among the 
numerous simulator designs, there are only a few including friction measurement (Bowsher and Shelton, 2001; Liao et al., 2003), which also provides very important data in the validation of numerical analysis of hip prostheses (Gao et al., 2018)

In this study, a new tribological hip joint simulator $\left(\mathrm{HSMS}_{4203}\right)$ with an improved friction measurement system is designed, built and validated. An online friction measuring module has been newly assembled in the hip joint simulator. Validation tests with UHMWPE and x-linked UHMWPE are performed and discussed. The purpose of this paper is to provide the features and development of this novel hip joint wear tester.

\section{MATERIALS AND METHODS}

\section{Apparatus Description}

The $\mathrm{HSMS}_{4203}$ applies similar principles with the biaxial simulator known as BRM. HSMS 4203 is capable of conducting the three motions that normal human hip joints do: Abduction and Adduction (AA), Flexion and Extension (FE), and Internal and External Rotation (IER). The main module is shown in Figure 1. The slope module is placed at the bottom, and the inclination is 23. The femoral head is connected to the slope module via a bearing housing, which holds a cross roller bearing. The distance between the head center and the slope plane is theoretically calculated and precisely assembled, so that the vertical loading axis can go through the head center as well as the rotation axis.

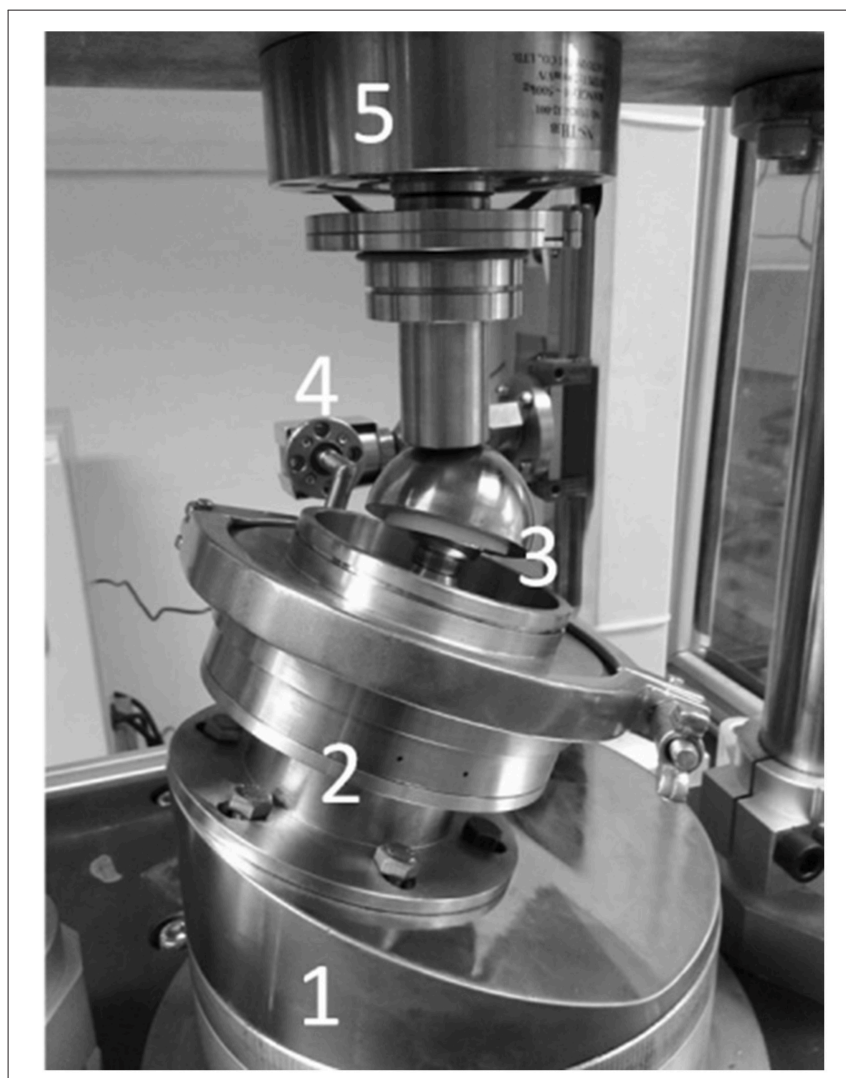

FIGURE 1 | The main module of the biotriboligical hip joint simulator (HSMS ${ }_{4203}$ ) without lubrication: (1) Slope module, (2) bearing housing, (3) reaction articulating surfaces, (4) friction measurement module, (5) force sensor.
The leaning shaft is a Z-shaped lever which will be described in the friction measurement part. A force sensor, connected in series with the loading axis, is placed above the reaction articulating surfaces and close to the soak control sample. The slide track and force track generated in the simulator have been discussed elsewhere (Hua and Zhang, 2008).

The newly developed friction measurement module is shown in Figure 2. The module is connected to the main module via the Z-shaped leaning lever (a), the axis of which goes through the center of the femoral head so that this axis is the AA axis. A bearing housing (b) then interlinks the lever and the senor (c), which makes the lever always vertical to the senor axis. The senor is bounded to the linear bearing $(\mathrm{d})$ by threaded and flange connection. The measuring range of the senor is 0 to $100 \mathrm{~N}$, and it is calibrated using weights. The rail of the linear bearing (d) is fixed to the simulator frame, in order to achieve an equatorial plane of the femoral head. Via this system, the friction force produced by the IER motion of the femoral head can be measured (Saikko, 2009).

Rotation frequency is controlled by a complemented frequency unit with a wide range from 0.2 to $5 \mathrm{~Hz}$. According to Johnston and Smidt (1969), young patients take more activities than the old ones. This indicates that the walking frequency may be one of the factors, which will have impact on bio-tribologicial of the hip prostheses. By using the frequency control unit, a different frequency rate can be set up to simulate various of walk speed.

\section{Validation Tests}

Two five-million-cycle-duration experiments were carried out. Commercial, artificial hip joints provided by one local hip prostheses manufacturer were used in the tests. The wear couples were $28 \mathrm{~mm}$ CoCrMo femoral heads against conventional and $\mathrm{x}$-linked UHMWPE acetabular cups. The internal diameter of the acetabular cups was $28 \mathrm{~mm}$. The cup was placed inside one metal shell. The metal shell locked the relative motion between the cup and itself. $2 \mathrm{kN}$ was vertically loaded on the metal shell by a pneumatic cylinder. The test frequency was $1 \mathrm{~Hz}$.

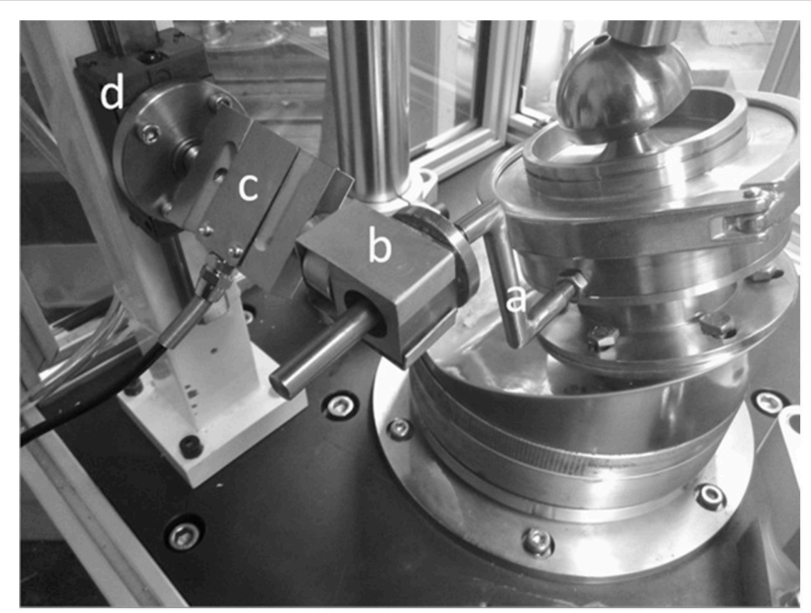

FIGURE 2 | Friction measurement module for $\mathrm{HSMS}_{4203}$ : (a) Z-shaped leaning lever, (b) bearing housing, (c) senor, (d) linear bearing and rail. 
The lubricant was Gibco Newborn Calf serum LOT1455368 and diluted 1:1 with deionized water. No additives were used. Evaporation was compensated with deionized water. Calf serum lubricant was replaced at every 1 million cycle.

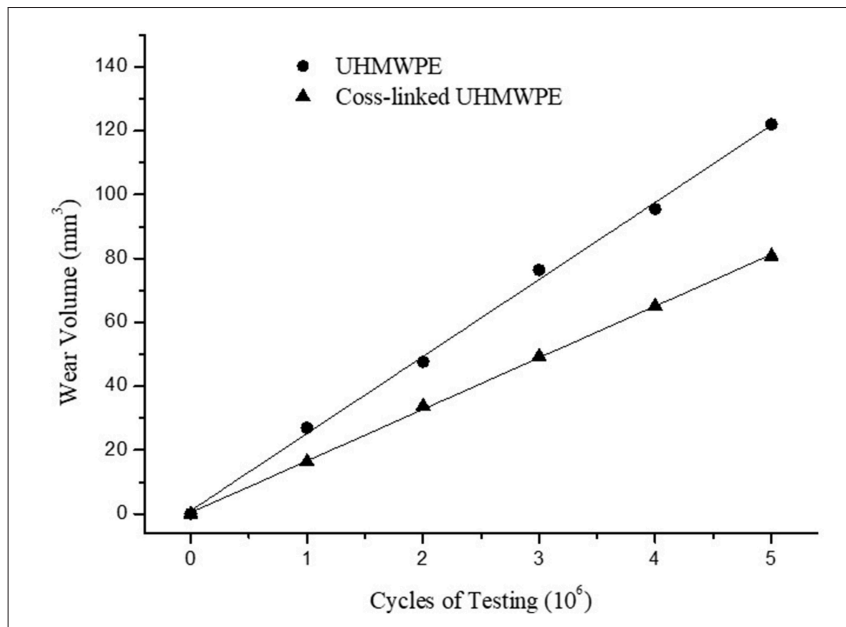

FIGURE 3 | Cumulative volumetric wear of UHMWPE and $x$-linked UHMWPE acetabular cups with cycles of testing. Results shown here are for a $28 \mathrm{~mm}$ head.
In the wear tests, the frictional force and loading were recorded continuously. The test was stopped every one million cycles, and the gravimetric wear was measured with a Sartorius balance $(0.01 \mathrm{mg})$, following an established procedure (ISO, 2016). The surface morphologies were observed by using Leica optical microscopy.

\section{RESULTS}

After more than 10 million cycles, the $\mathrm{HSMS}_{4203}$ ran smoothly and no malfunction occurred. Cumulative gravimetric wear for the two samples (UHMWPE and $\mathrm{x}$-linked UHMWPE acetabular cups) exhibited a linear relationship with an increase in the number of motion cycles. Volumetric wear per cycle $\left(\mathrm{mm}^{3} /\right.$ cycle) was therefore calculated and shown in Figure 3. The correlation coefficients of linear regression of the mean volumetric wear values were 0.99757 (UHMWPE), and 0.99953 (x-linked UHMWPE). The slopes of the linear regression, also known as the wear rates, were $24 \mathrm{~mm}^{3} / 10^{6}$ of UHMWPE, and $16 \mathrm{~mm}^{3} / 10^{6}$ of $\mathrm{x}$-linked UHMWPE. The initial two samples' morphologies are shown in Figures 4A,B, while the wear surfaces after 5 million cycles are shown in Figures 4C,D.

Continuous dynamic friction was acquired by the senor in the friction measurement module. Except for the running-in effect at

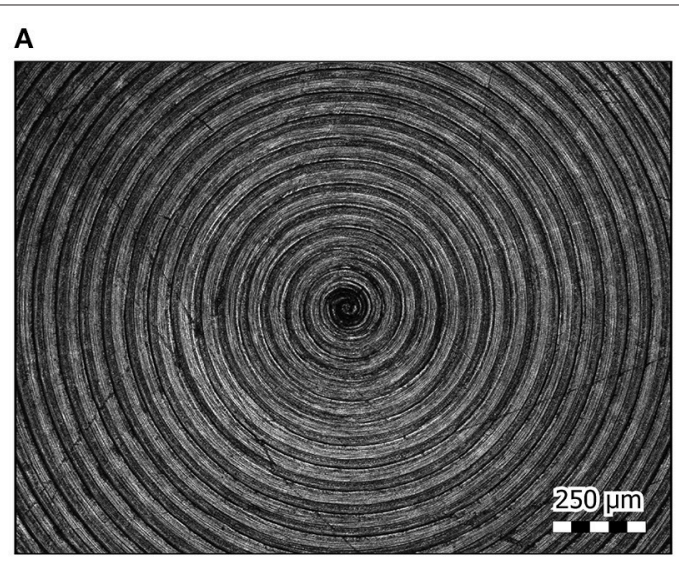

C

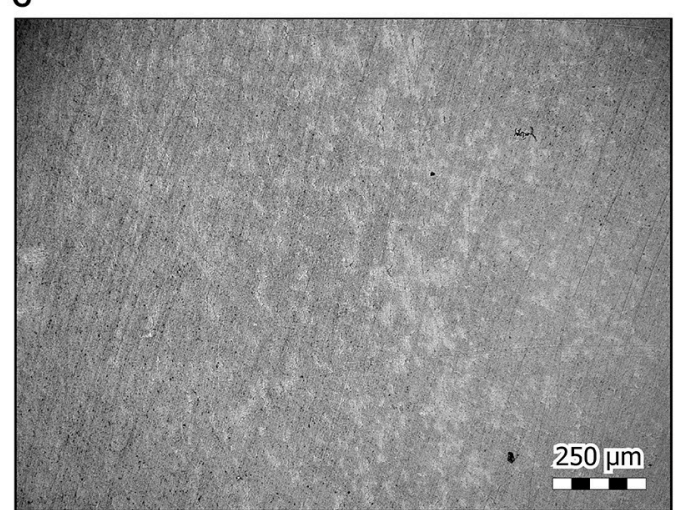

B

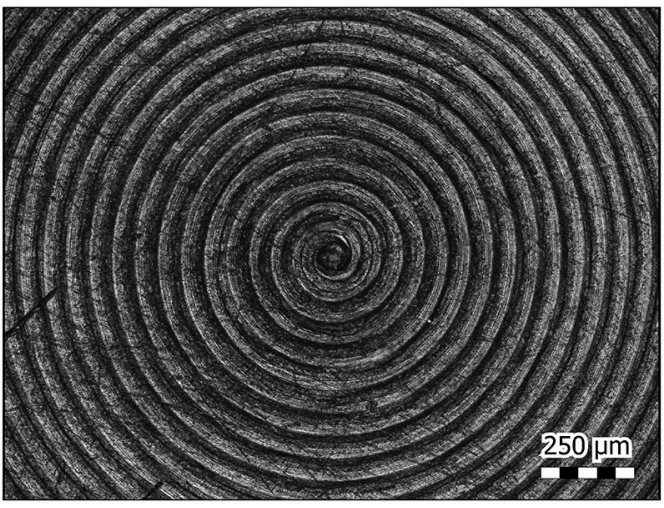

D

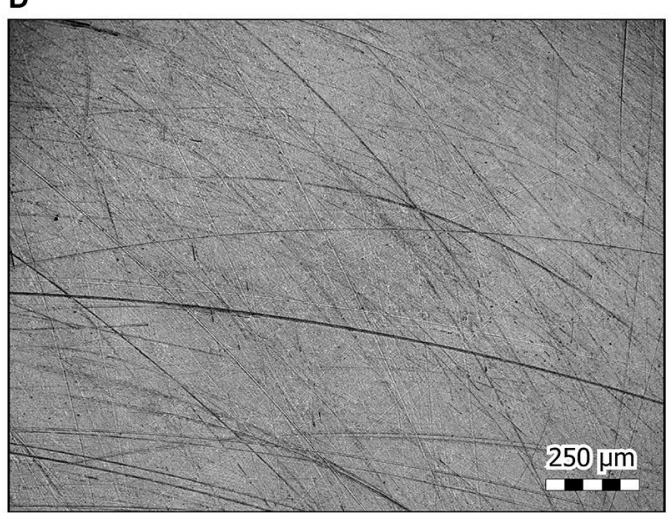

FIGURE 4 | Optical micrographs of the cup wear surfaces (A) original UHMWPE, (B) original x-linked UHMWPE (C) UHMWPE after 5 million cycles, (D) x-linked UHMWPE after 5 million cycles. 
every start, most data were stable and showed periodic variation with two maxima and two minima per cycle (Figure 5). The maxima were observed when the upper part of the Z-shaped lever is at the horizontal positions, and the minima were at the top and bottom positions. Also, the frictional force can be distinguished from the two tested samples. For the UHMWPE acetabular cups, they vary from $0 \sim 32 \mathrm{~N}$ to $0 \sim 28 \mathrm{~N}$ for the $\mathrm{x}$-linked one.

\section{DISCUSSION}

The duration of an ISO14242 standard wear test for artificial hip joints is 5 million cycles (ISO, 2014). Therefore, the reliability of the hip joint simulator is very important so as to achieve a non-stop testing condition for 1 million cycles at least. The $\mathrm{HSMS}_{4203}$ was still in healthy condition after 10 million cycles. This confirmed that the strong reliability requirement of the simulator could be met. In some retrieval analysis of artificial joints, the clinical volumetric wear is of the order of $10 \mathrm{~mm}^{3} / 10^{6}$ cycles (Lachiewicz et al., 2009; Wang et al., 2013; Hua et al., 2014). Herein, the volumetric wear loss of conventional UHMWPE (24 $\left.\mathrm{mm}^{3} / 10^{6}\right)$ and $\mathrm{x}$-linked UHMWPE $\left(16 \mathrm{~mm}^{3} / 10^{6}\right)$ are within the range. This indicates that the clinical wear of artificial hip joints could be reperformed in vitro by the $\mathrm{HSMS}_{4203}$.

The linearity of wear rates on the testing cycles is highly dependent (Figure 3). This indicates that this method can provide a good prediction of the wear loss. The wear rate of $\mathrm{x}$-linked UHMWPE is almost $60 \%$ of UHMWPE. In wear testing of orthopedic implants, a reliable method requires the capability to distinguish between original design purpose and obtain a clear comparison. It was found that the difference in the tribological performance of artificial hip joints can be distinguished in $\mathrm{HSMS}_{4203}$, even between conventional and modified biomaterials, i.e., UHMWPE. Furthermore, it was also found that the volumetric wear of the x-linked UHMWPE acetabular cup is lower than the normal UHMWPE acetabular cup at the product level, which is in accordance with the wear tested at material level (Hua et al., 2014). As shown in Figure 4, after 5 million cycles test, the UHMWPE acetabular cup's wear surfaces are burnished, which are typical surface morphologies under multi-direction hip motion (Saikko, 2005). The surface of the $\mathrm{x}$-linked UHMWPE cup is not as well-burnished, but more cross scratches have been observed, meaning that less material is worn out, referring to its lower wear rate.

For the whole test period, the frictional force tends to be stable, except for every start after the interval. It can be two times larger than the stable value. The cause of this phenomenon could be that at the start the head center is not self-centered yet. So if the slide track is deviated, even edge-effects can exist, making the loading pressure vibrate or go up sharply. Another possible cause could be that after the running-in period, the friction pair is burnished, and therefore, can form better lubrication conditions.

The frictional force in stable period is of the same order as Saikko (2009) and can be obviously distinguished between the two tested samples. The frictional force of the $\mathrm{x}$-linked UHMWPE acetabular cup is nearly $10 \%$ lower than the UHMWPE's, which shows that the trend is in accordance with the volumetric wear, making the new friction measurement module

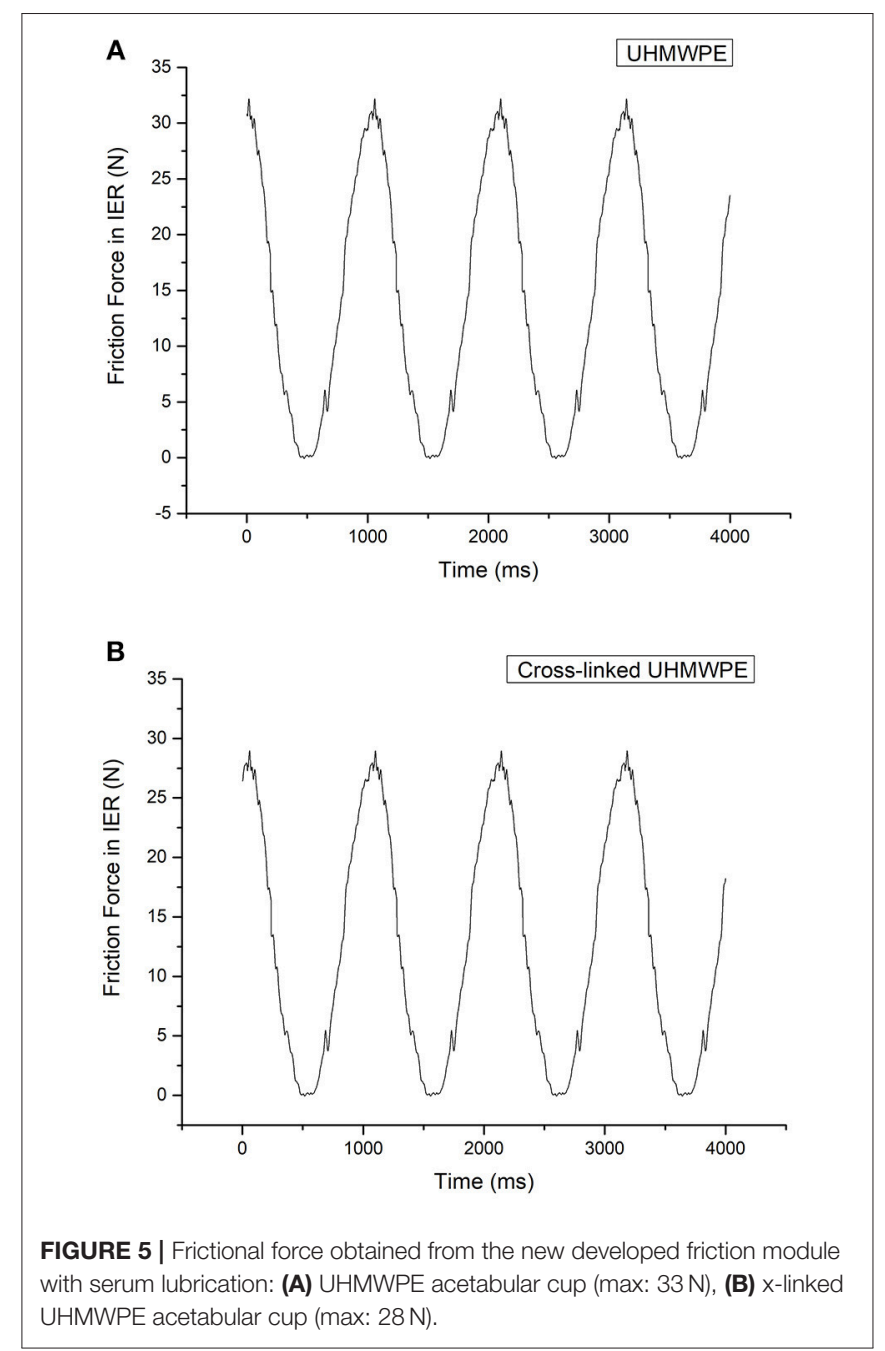

valid for the biotribological investigation of hip prostheses. Correspondingly, friction of the implant product proves to be a key factor affecting the wear performance in joint articulation.

In future work, more studies can be carried out via this newly developed hip joint simulator $\left(\mathrm{HSMS}_{4203}\right)$. The new lubricants, such as bionic joint fluids (Hua et al., 2007), could be evaluated both for friction and wear under a specified duration, under body-simulated conditions. Moreover, product-level tests for some advanced surface technologies aimed at decreasing friction in hip joints, such as super-lubrication technology (Jiao et al., 2018), could be performed.

\section{CONCLUSION}

A new wear test apparatus, hip joint simulator $\left(\mathrm{HSMS}_{4203}\right)$ has been developed for the biotribological investigation of artificial hip joints. The $\mathrm{HSMS}_{4203}$ is in healthy condition after 10-million-cycle tests. The highly linear wear rates observed in the experiments agree well with the clinical results. The difference in wear performance of artificial hip wear couples (CoCrMo on UHMWPE and CoCrMo on $\mathrm{x}$-linked UHMWPE) can be distinguished. Moreover, the $\mathrm{HSMS}_{4203}$ provides a potential solution toward the 
friction monitoring to obtain more dynamic data in artificial joint wear tests.

\section{AUTHOR CONTRIBUTIONS}

$\mathrm{ZH}$ corresponding author, who has designed the hip simulator. $\mathrm{HJ}$ codesigner of the hip simulator. FT performed the experiment. XW and XX instructed the experiments in the

\section{REFERENCES}

Bowsher, J. G., and Shelton, J. C. (2001). A hip simulator study of the influence of patient activity level on the wear of crosslinked polyethylene under smooth and roughened femoral conditions. Wear 250, 167-179. doi: 10.1016/S0043-1648(01)00619-6

Cheng, G., and Shan, X. (2012). Dynamics analysis of a parallel hip joint simulator with four degree of freedoms (3R1T). Nonlin. Dynam. 70, 2475-2486. doi: 10.1007/s11071-012-0635-4

Chu, C. R. (2015). short-term analysis vs long-term data on total hip replacement survivorship. JAMA Surg.150, 989-989. doi: 10.1001/jamasurg. 2015.1337

Ferguson, R. J., Palmer, A., Taylor, A., Porter, M. L., Malchau, H., and Sion GlynJones, S. (2018). Hip and knee replacement 1: hip replacement. Lancet 392, 1662-1671. doi: 10.1016/S0140-6736(18)31777-X

Gao, L., Hua, Z. K., and Hewson, R. (2018). Can a "pre-worn" bearing surface geometry reduce the wear of metal-on-metal hip replacements? - A numerical wear simulation study. Wear 406-407, 13-21. doi: 10.1016/j.wear.2018. 03.010

Hua, Z., Zhang, H., Fan, Y., and Jin, Z. (2014). Development of a BiotriboPOD testing methodology for the wear evaluation of orthopaedic biomaterials. RSC Adv. 4, 19987-19991. doi: 10.1039/C4RA01743A

Hua, Z. K., and Zhang, J. H. (2008). A new simulator for bio-tribological study. J. Bionic Eng. 5, 143-147. doi: 10.1016/S1672-6529(08) 60085-2

Hua, Z. K., Zhang, J. H., and Su, S. H. (2007). Tribological study on the bionic therapeutic fluids. Tribol. Lett. 28, 51-58. doi: 10.1007/s11249-007-9247-4

ISO, (2009). ISO 14242-3:2009. Implants for Surgery - Wear of Total Hip-Joint Prostheses - Part 3: Loading and Displacement Parameters for Orbital Bearing Type Wear Testing Machines and Corresponding Environmental Conditions for Test. ISO.

ISO, (2014). ISO 14242-1.2014. Implants for Surgery - Wear of Total Hip-Joint Prostheses - Part 1: Loading and Displacement Parameters for Wear-Testing Machines and Corresponding Environmental Conditions for Test. ISO.

ISO, (2016). ISO 14242-2.2016. Implants for Surgery - Wear of Total Hip-Joint Prostheses - Part 2: Methods of Measurement. ISO.

Jiao, Y., Liu, S., Sun, Y. , Yue, W., and Zhang, H. (2018). Bioinspired surface functionalization of nanodiamonds for enhanced lubrication. Langmuir 34, 12436-12444. doi: 10.1021/acs.langmuir.8b02441 manuscript. LG and $\mathrm{XH}$ instructed the friction measuring in the experiment. ZJ instructed the design of hip simulator. PD co-performed of the validation test and did the wear and friction measurement.

\section{ACKNOWLEDGMENTS}

Natural Science Foundation of Shanghai (16ZR1411800).

Johnston, R. C., and Smidt, G. L. (1969). Measurement of hip-joint motion during walking. J. Bone Joint Surg Am. 51, 1083-1094. doi: 10.2106/00004623-196951060-00003

Lachiewicz, P. F., Heckman, D. S., Soileau, E. S., Mangla, J., and Martell, J. M. (2009). Femoral head size and wear of highly $\mathrm{x}$-linked polyethylene at 5 to 8 years. Clin. Orthop. Relat. R. 467, 3290-3296. doi: 10.1007/s11999-009-1038-9

Liao, Y. S., McKellop, H., Lu, Z., Campbell, P., and Benya, P. (2003). The effect of frictional heating and forced cooling on the serum lubricant and wear of UHMW polyethylene cups against cobalt-chromium and zirconia balls. Biomaterials 24, 3047-3059. doi: 10.1016/S0142-9612(03)00148-0

McKellop, H. A., Campbell, P., Park, S. H., Schmalzried, T. P., Grigoris, P., Amstutz, H. C., et al. (1995). The origin of submicron polyethylene wear debris in total hip arthroplasty. Clin. Orthop. Relat. R. 311, 3-20

Oliveira, A. L. L., Trigo, F. C., Queiroz, R. D., and Carvalho, R. T. (2013). Development of a protocol for the performance evaluation of wear machines used in tests of joint prostheses. Mech. Mach. Theory 61, 59-67. doi: 10.1016/j.mechmachtheory.2012.10.011

Saikko, V. (2005). A 12-station anatomic hip joint simulator. Proc. Inst. Mech. Eng. 219, 437-448. doi: 10.1243/095441105X34419

Saikko, V. (2009). Friction measurement in the biaxial rocking motion hip joint simulator. J. Tribol. 131:011201. doi: 10.1115/1.2991121

Saikko, V., and Shen, M. (2010). Wear comparison between a dual mobility total hip prosthesis and a typical modular design using a hip joint simulator. Wear 268, 617-621. doi: 10.1016/j.wear.2009.10.011

Wang, A., Lee, R., Herrera, L., and Korduba, L. (2013). Wear of ultra-high molecular weight polyethylene moving along a circular path in a hip simulator. Wear 301, 157-161. doi: 10.1016/j.wear.2012.11.023

Conflict of Interest Statement: The authors declare that the research was conducted in the absence of any commercial or financial relationships that could be construed as a potential conflict of interest.

Copyright (C) 2019 Hua, Dou, Jia, Tang, Wang, Xiong, Gao, Huang and Jin. This is an open-access article distributed under the terms of the Creative Commons Attribution License (CC BY). The use, distribution or reproduction in other forums is permitted, provided the original author(s) and the copyright owner(s) are credited and that the original publication in this journal is cited, in accordance with accepted academic practice. No use, distribution or reproduction is permitted which does not comply with these terms. 